\title{
Terapias alternativas/complementares: o saber e o fazer das enfermeiras do distrito administrativo 71 - Santo Amaro - São Paulo*
}

\author{
ALTERNATIVE OR COMPLEMENTARY THERAPIES: KNOWLEDGE AND ACTION OF THE NURSES AT THE \\ 71stADMINISTRATIVE DISTRICT - SANTO AMARO, SÃO PAULO, BRAZIL \\ TERÁPIAS ALTERNATIVAS /COMPLEMENTARIAS: EL SABER Y EL HACER DE LAS ENFERMERAS DEL \\ DISTRITO ADMINISTRATIVO n. 71 - SANTO AMARO- SAN PAULO-BRASIL
}

Helena Maria Fekete Nuñez ${ }^{1}$, Suely Itsuko Ciosak ${ }^{2}$

\section{RESUMO}

Este estudo objetivou verificar o saber e o fazer das enfermeiras que atuam nas unidades municipais de Saúde do Distrito Administrativo 71-Santo Amaro-São Paulo, frente às Terapias Alternativas/ Complementares $(T A / C)$. A pesquisa, de abordagem quantitativa com análise qualitativa dos dados, permitiu evidenciar que $89 \%$ dos enfermeiros acreditam nas $T A / C$ porém, apenas 22,2\% têm conhecimento do respaldo legal e 5,5\% têm cursos nesta área; 44,4\% aplicam em si mesma e 11,1\% aplicam nos pacientes, sete TA/C distintas, classificadas em cinco grupos de HILL. Desvendou-se que há necessidade de buscar novos saberes como opções de assistência à promoção da saúde da população.

\footnotetext{
PALAVRAS-CHAVE

Enfermagem holística. Terapias alternativas. Terapias complementares. Promoção da saúde. Pesquisa em enfermagem.
}

\author{
ABSTRACT \\ The purpose of this study is \\ to investigate the nurses \\ knowledge and actions taken \\ regarding alternative or \\ complementary therapies \\ (AT or CT) at the County \\ (municipal) Health Units of \\ the $71^{\text {st }}$ Administrative \\ District, in Santo Amaro, SP. \\ The research use a \\ quantitative aproach and $a$ \\ qualitative analysis of the field \\ data that showed that $89 \%$ of \\ the nurses have confidence in \\ AT/CTs but only $22,2 \%$ know \\ the legal basis for its use and \\ $5,5 \%$ had courses on the \\ techniques. $44,4 \%$ use these \\ therapies on themselves and \\ $11,1 \%$ on their patients. \\ Altogether 7 diferent AT/CTS \\ were listed and classified in \\ 5 Hill's groups. \\ It was found that there is a \\ need for including new \\ knowledge as a \\ complementary option for \\ the promotion of the \\ population's health.
}

\section{KEYWORDS}

Holistic nursing.

Alternative therapies.

Complementary therapies.

Health promotion.

Research on nursing.

\section{RESUMEN}

Este estudio tuvo por objetivo verificar el saber y el hacer de las enfermeras que trabajan en las unidades municipales de Salud del Distrito Administrativo n. 71, de Santo Amaro -San Paulo Brasil, en relación a las Terapias Alternativas/ Complementarias (TA/C). La investigación, de abordaje cuantitativo con análisis cualitativo de los datos, demonstró que el $89 \%$ de las enferrmeras creen en las TA/C, sin embargo apenas $22,2 \%$ tienen conocimiento del fundamento legal y 5,5\% hicieron cursos en esta area; 44,4\% aplican en sí mismas y $11,1 \%$ aplican en los pacientes siete TA/C distintas, clasificadas en cinco grupos de HILL. Se constató la necesidad de buscar nuevos conocimientos como opciones de asistencia para la promoción de la salud de la población.

\section{PALABRAS-CLAVE}

Enfermería holística.

Terápias alternativas;

Terápias complementares;

Promoción de la salud;

Investigación en enfermeria.
* Trabalho extraído da Dissertação de Mestrado em Enfermagem intitulada "Terapias alternativas/ complementares: o saber e o fazer das enfermeiras do Distrito Administrativo 71-

Santo Amaro - São

Paulo" do Programa de Pós-Graduação em Enfermagem de Saúde Coletiva-Escola de Enfermagem da USP (EEUSP), 2002.

1 Enfermeira do Programa da Saúde da Família, Jd Monte Azul, Vila das Belezas, DS São Luiz, São Paulo; Mestre em Enfermagem na área de Saúde Coletiva (EEUSP).

2 Profa Dra do Departamento de Enfermagem em Saúde Coletiva da EEUSP; Diretora do Departamento de Enfermagem do Hospital Real e Benemérito da Sociedade Portuguesa de Beneficência. 


\section{INTRODUÇÃO}

Na época da minha formação profissional, na década de setenta, pouco se falava sobre tratamentos alternativos à medicina tradicional, também conhecidos como terapias alternativas ou complementares ( TA/C).

Após muitos anos de experiência na enfermagem alopática, em 1998, prestei serviços de enfermagem numa clínica formada por uma equipe multidisciplinar, onde os pacientes, na grande maioria oncológicos, ou portadores de doenças imunológicas, recebiam além de tratamentos convencionais, tratamentos complementares como: homeopáticos, antroposóficos, fitoterápicos, imunoterápicos, toque terapêutico, Reiki, desbloqueio e alinhamento dos chakras, terapia artística, euritmia curativa, relaxamento, visualização, troca de vivência em grupo, meditação e trabalho biográfico antroposófico, que visavam torná-los mais conscientes e colaboradores na promoção, prevenção e recuperação da sua saúde.

Percebi que com esta atuação mais integral, no cuidar, o paciente relatava grande melhora na qualidade de vida durante seu processo de doença.

\begin{abstract}
Silva, Gimenes ${ }^{(1)}$ afirmam que
o paradigma emergente, também chamado de holístico (etimologicamente, holístico e holismo derivam do grego holikós, que significa todo, inteiro, completo e na filosofia é a síntese de unidades em totalidades organizadas), evita tratar de forma isolada o processo saúde-doença, que tende a ser visualizado com um continuum, em que a saúde deixa de ser um estado estático de perfeito bem estar, subentendendo-se mudança contínua aos desafios ambientais e ao equilíbrio dinâmico do organismo, que envolve aspectos físicos, psicológicos, mentais, sociais e espirituais.
\end{abstract}

Nas Teorias que fundamentam a Enfermagem, de Martha Rogers e Myra E. Levine, o Homem é visto como um ser holístico ${ }^{(2)}$.

Com a expansão do modelo mecanicista newtoniano da Física pela visão einsteiniana da Física Quântica, os seres humanos passam a ser vistos não mais apenas como máquinas ${ }^{(3,4)}$ e sim, como campos de energia que se interpenetram e se influenciam reciprocamente. Com isso, Gerber ${ }^{(3)}$ introduziu na medicina convencional um sistema de pensamentos que estuda o funcionamento corpo humano a partir de uma perspectiva que considera ser ele constituído por múltiplos sistemas energéticos que se influenciam reciprocamente e que nossos pensamentos e emoções afetam nossa fisiologia e que terapias simples, como ervas, água, essências florais, por exemplo, podem ser agentes de cura muito eficazes.

É baseado neste conceito que as TA/C são consideradas, pois elas podem ser definidas como

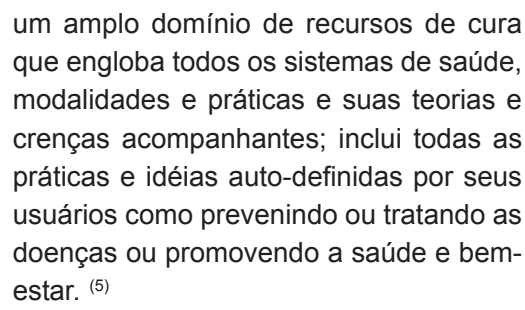

Nesta pesquisa foram adotados os termos terapias alternativas e terapias complementares, indicando a mesma prática, embora não sejam sinônimos, mas porque o termo alternativas é o mais divulgado.

Na organização de Hill( ${ }^{(6)}$, as correntes terapêuticas básicas das medicinas alternativas são classificadas em quatro: Medicina Oriental, Hindu ou Ayurvédica, Homeopática e a Medicina ampliada pela Antroposofia e as técnicas ou práticas complementares nem sempre pertencem apenas a uma corrente de medicina alternativa/complementar; por exemplo, tanto a medicina chinesa quanto ayurvédica, homeopática e antroposófica, fazem uso da fitoterapia, mas cada uma com o enfoque de sua corrente. A população, que faz uso das TA/C, procura em geral as práticas que lhe fazem bem, não se importando na maioria dos casos em seguir uma determinada linha de medicina. A autora, classifica as TA/C em sete grupos distintos: terapias físicas, hidroterapias, fitoterapias, terapias nutricionais, terapias mentais-espirituais, e terapias de exercícios individuais.

Existem ainda outras terapias que não são mencionadas por Hill( ${ }^{(6)}$, porém são relatadas por muitos profissionais da saúde e terapêutas, tais como a Calatonia, Cura Prânica, Reiki, Toque Terapêutico, Neurolingüística, Terapia Biográfica Antroposófica, Tai-Chi-Chuan, LianGong, Do-In. 
No Brasil, podemos destacar como enfermeiras pioneiras nos estudos na área de TA/ C, Dra Maria Jacyra Nogueira (7) e Dra Maria Alves Barbosa ${ }^{(8)}$.

Para sedimentar as TA/C, o próprio COFEN apoiou, através do Parecer Normativo $n^{\circ} 004$ / 95, o reconhecimento das práticas alternativas (Acupuntura, Iridologia, Fitoterapia, Reflexologia, Quiropraxia, Massoterapia, dentre outras), como atividade profissional vinculada à saúde e não estando vinculados a qualquer categoria profissional; e através da Resolução COFEN-197/97 que

estabelece e reconhece as Terapias Alternativas como especialidade e/ou qualificação do profissional de Enfermagem, desde que o profissional de Enfermagem conclua e tenha sido aprovado em curso reconhecido por instituição de ensino ou entidade congênere, com uma carga horária mínima de 360 horas ${ }^{(9)}$.

Segundo Antunes ${ }^{(10)}$,

a implantação do Sistema Único de Saúde no Brasil, trouxe um grande desafio para a enfermagem: redirecionar suas práticas para o atendimento integral à saúde coletiva e individual da população brasileira,

o que poderia ser amparada pelas TA/C, porém a falta de uma pesquisa voltada à atenção primária à saúde com as $\mathrm{TA} / \mathrm{C}$, levou à presente investigação, cujo objetivo principal é verificar o saber e o fazer das enfermeiras, que atuam nas unidades de saúde do Dis- trito Administrativo-71 Santo Amaro, do Município de São Paulo, sobre as TA/C, e os objetivos específicos são: caracterizar as enfermeiras que atuam nas unidades de saúde, junto à população, identificar quais são as TA/C conhecidas e utilizadas pelas enfermeiras, verificar os resultados obtidos com as $\mathrm{TA} / \mathrm{C}$, as facilidades e dificuldades encontradas pelas enfermeiras, nas Unidades de Saú$\mathrm{de}$, para exercerem as TA/C.

\section{MATERIAL E MÉTODOS}

O projeto recebeu parecer favorável do Comitê de Ética em Pesquisa (CEP/EEUSP), sob o parecer n. 148/2001.

Após autorização escrita de cada diretor das nove unidades de saúde, foi solicitando anuência das(os) enfermeiras(os), através do consentimento livre, em participar do presente estudo, sendo assegurado o anonimato.

Foi realizado um estudo exploratório prospectivo, sobre o conhecimento e a prática que as enfermeiras têm sobre as TA/C, através da aplicação de um questionário à todas as enfermeiras que atuam diretamente com usários, nas unidades municipais de saúde do Distrito Administrativo-71 (DA-71) Santo Amaro.

Os nove Serviços de Saúde do DA Santo Amaro que possuem enfermeiras e atendem diretamente a população em assistência primária e secundária, e que são foco desta investigação, encontram-se na Tabela 1, a seguir:

Tabela 1 - Relação da Unidades de Saúde do DA -71 e total de enfermeiras lotadas nas respectivas unidades (São Paulo-jun/ago- 2001)

\begin{tabular}{|c|c|c|c|}
\hline $\begin{array}{l}\text { Tipo de } \\
\text { US }\end{array}$ & Unidade de Saúde & Enderego & $\begin{array}{l}\text { Enfermeiras } \\
\text { por unidade }\end{array}$ \\
\hline UBS & $\begin{array}{l}\text { PAM Santo Amaro } \\
\text { Paulo Eiró } \\
\text { UBS Chácara } \\
\text { Santo Antonio }\end{array}$ & $\begin{array}{l}\text { Rua Paulo Eiró, 19/23-Santo Amaro } \\
\text { Tel.: } 5548-6636 \\
\text { Rua Alexandre Dumas, } 719 \\
\text { Chácara Sto. Antônio / Tel.: } 5181-7894\end{array}$ & $\begin{array}{l}2 \\
2\end{array}$ \\
\hline CR & $\begin{array}{l}\text { CR DST/AIDS } \\
\text { Santo Amaro } \\
\text { Unidade de } \\
\text { Farmacodependência } \\
\text { CR. Saúde do } \\
\text { Trabalhador }\end{array}$ & $\begin{array}{l}\text { R. Gal. Roberto A. de Carvalho Fo, } 569 \\
\text { Tel.: 5523-6052 } \\
\text { Rua Adolfo Pinheiro, } 1463 \text { Sto. Amaro } \\
\text { Tel.: } 5523-3566 \\
\text { Av. Adolfo Pinheiro, } 581 \text { Sto. Amaro } \\
\text { Tel.: 5523-5382 }\end{array}$ & $\begin{array}{l}2 \\
1 \\
3\end{array}$ \\
\hline $\mathrm{CO}$ & COAS Santo Amaro & $\begin{array}{l}\text { Rua Prof. Gabriel Netuzzi Perez, } 159 \\
\text { Sto. Amaro / Tel.: } 246-9960\end{array}$ & 1 \\
\hline HD & $\begin{array}{l}\text { Amb. Saúde } \\
\text { Mental Infantil } \\
\text { Amb. Saúde Mental } \\
\text { Santo Amaro }\end{array}$ & $\begin{array}{l}\text { Rua Sena Milton Campos, } 149 \\
\text { Chácara Sto. Antônio / Tel.: 5181-3114 } \\
\text { Rua Paula Cruz, } 71 \\
\text { Tel.: } 246-6076\end{array}$ & $\begin{array}{l}1 \\
1\end{array}$ \\
\hline $\mathbf{A E}$ & $\begin{array}{l}\text { Amb. de Especialidades } \\
\text { doHosp. Reg. Sul (ARES) }\end{array}$ & $\begin{array}{l}\text { Av. Adolfo Pinheiro, } 122 \text { Sto. Amaro } \\
\text { Tel.: } 5521-0088\end{array}$ & 5 \\
\hline TOTAL & 9 & & 18 \\
\hline
\end{tabular}

Terapias alternativas/ complementares: o saber e o fazer das enfermeiras do

Distrito Administrativo 71 - Santo Amaro São Paulo 
Helena Maria F. Nuñez

Suely Itsuko Ciosak

\section{RESULTADOS}

Percorrendo as nove Unidades de Saúde do DA-71 Santo Amaro, foram entrevistadas 18 enfermeiras, que correspondem ao total dos profissionais destas unidades, sendo $17(94,4 \%)$ do sexo feminino e $1(5,6 \%)$, do sexo masculino. Os mesmos resultados foram também constatados por Bezerra ${ }^{(11)}$, Oguisso $^{(12,13)}$ e Ciosak ${ }^{(14)}$, confirmando mais uma vez que esta profissão continua sendo predominantemente feminina (cerca de 95\%).

Como algumas práticas religiosas ou filosofias de vida condenam ou reforçam algu- mas práticas alternativas, buscou-se entre as entrevistadas conhecer esta prática e verificamos que embora $15(83,3 \%)$ enfermeiras referem ter uma filosofia de vida ou uma religião, existe uma grande heterogeneidade quanto à sua prática: $4(22,2 \%)$ enfermeiras são Católicas; 3(16,7\%) são Adventistas e 3 Kardecistas. Também foram referidas o Budismo, Messiânica, Batista e Protestante, a filosofia de vida de "amar ao próximo", mostrando existir oito diferentes religiões ou filosofias e um ateísmo entre as enfermeiras entrevistadas; 2 (11,1\%) não responderam, neste quesito (Quadro 1).

Quadro 1 - Relação das Religiões, Escolas de Graduação e Pós-Graduações das Enfermeiras do DA-71 - (São Paulo - 2001)

\begin{tabular}{|c|c|c|c|c|c|c|c|c|c|}
\hline $\begin{array}{l}\text { Religiōes ou } \\
\text { Filosofia de } \\
\text { Vida }\end{array}$ & $\mathbf{N}$ & $\%$ & & $\begin{array}{l}\text { ESCOLAS DE } \\
\text { GRADUAÇÃO }^{* *}\end{array}$ & $\mathbf{N}$ & $\%$ & $\begin{array}{l}\text { PósGRADUAÇÕE } \\
\text { CURSADAS }\end{array}$ & $\mathbf{N}^{*}$ & $\%$ \\
\hline Católica & 4 & 22,2 & EEPr & Fac. Adventista de Enf. -SP cap. & 6 & 33,3 & Mestrado & 1 & 5,6 \\
\hline Adventista & 3 & 16,7 & EEPr & Escola de Enf. São José -SP cap. & 2 & 11,1 & Adm. Hospit. & 5 & 27,7 \\
\hline Kardecista & 3 & 16,7 & EEPú & Escola de Enf. da USP -SP cap. & 2 & 11,1 & Gerenc. Enf. & 1 & 5,6 \\
\hline Protestante & 1 & 5,6 & EEPr & Fac. de Enf São Camilo -SP cap. & 1 & 5,6 & Esp.S.Públic. & 3 & 16,7 \\
\hline Budista & 1 & 5,6 & EEPr & Fac. Integr. de Guarulhos-SP cap. & 1 & 5,6 & Hab.S.Públic. & 2 & 11,1 \\
\hline Messianica & 1 & 5,6 & EEPr & Fac, de Enf. Barão de Mauá -SP & 1 & 5,6 & Enf.do Trab. & 2 & 11,1 \\
\hline Batista & 1 & 5,6 & EEPú & int. & 1 & 5,6 & Educ.S.Públic. & 1 & 5,6 \\
\hline Outras & 1 & 5,6 & $\mathrm{EEPr}$ & Univ.Fed. de Juiz de Fora-MG & 1 & 5,6 & Hab.Obstetr. & 4 & 22,2 \\
\hline ateísmo & 1 & 5,6 & EEPú & Esc. de Enf. Wenceslau Brás -MG & 1 & 5,6 & Licenciatura & 2 & 11,1 \\
\hline \multirow[t]{6}{*}{ Nenhuma } & 2 & 11,1 & EEPú & Univ. Fed, da Bahia-BA & 1 & 5,6 & Estomaterap. & 2 & 11,1 \\
\hline & & & EEPú & Univ. Fed. da Paraíba -PB & 1 & 5,6 & Hab.M.Cirúrg. & 1 & 5,6 \\
\hline & & & & Univ. Fed. do Pará -PA & & & Psiquiatria & 1 & 5,6 \\
\hline & & & & & & & Nefrologia & 1 & 5,6 \\
\hline & & & & & & & CCIH & 1 & 5,6 \\
\hline & & & & & & & Nenhuma & 4 & 22,2 \\
\hline
\end{tabular}

**Escolas de Graduação: abreviaturas:

$\mathrm{N}^{*}$ é o número de enfermerias

Fez-se um levantamento das Escolas de Enfermagem, buscando, ainda, apreender a influência da formação no conhecimento, percepção e aceitação das TA/C; as Escolas de Enfermagem foram classificadas em públicas (EEPú) e privadas ( EEPr) (Quadro 1).

As enfermeiras se graduaram em 11 diferentes Escolas de Enfermagem das quais 5(45,4\%) são EEPú, e 6(54,6\%) são EEPr; a maioria da enfermeiras, $12(66,7 \%)$ é oriunda de EEPr e 6 (33,3\%) de EEPú (Quadro 1).

Foi realizado, também, um levantamento sobre as Pós Graduações (PG) cursadas pelas enfermeiras, para averiguar quais os cursos ou tendências que as enfermeiras buscaram, para complementar sua formação básica e se algumas já demonstravam interesse nas áreas de TA/C. Verificou-se que as enfermeiras do DA-71, frequentaram um total de 27 cursos de PG, sendo 23(85\%) cursadas pelas enfermerias de EEPr. Chama atenção, ainda, que nenhuma das enfermeiras entrevistadas cursou PG em TA/C (Quadro 1).

Das enfermeiras do DA-71, 17(94,4\%) tem mais de 35 anos de idade e $8(44,4 \%)$ tem de 15 a 20 anos de formada, sugerindo estar no mercado de trabalho há muitos anos, confirmados pelos dados seguintes: $13(72,2 \%)$ enfermeiras tem mais de dez anos de trabalho no Serviço Público, revelando ser um grupo de enfermeiras estável no sistema de trabalho, acrescentando assim ao saber acadêmico, um saber enriquecido pela prática da profissão. Das 18 enfermeiras, 11(61,1\%), atuam há menos de cinco anos na unidades atuais.

Quanto ao modo como as enfermeiras percebem e reagem frente às TA/C, 16(89\%) acreditam nas TA/C, mostrando não haver interferência da religião nem da formação acadêmica e $8(44,4 \%)$ têm experiência na área(Figura 1). 


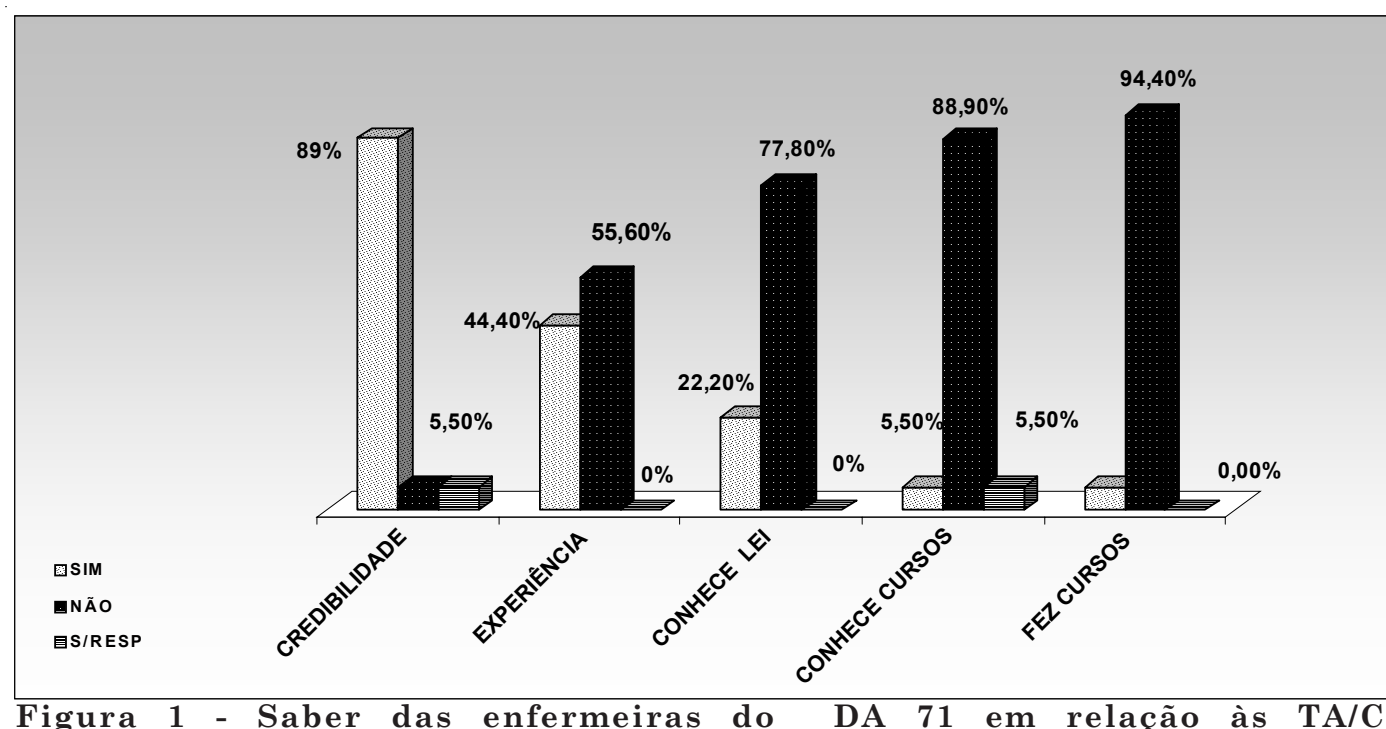

Terapias alternativas/ complementares:

o saber e o fazer das enfermeiras do

Distrito Administrativo

71 - Santo Amaro -

São Paulo

Chama a atenção o fato de apesar do COREN ter promulgada a Resolução COFEN197 em 1997, $14(77,8 \%)$ do total das enfermeiras referem não conhecer o respaldo legal e 16 (89\%) não conhecem cursos que as tornariam especialistas em TA/C. Somente um profissional disse ter feito curso na área de $\mathrm{TA} / \mathrm{C}$, com carga horária de $20 \mathrm{hs}$, durante a graduação (FAE) ( Figura 1).

Podemos constatar na população desta pesquisa, que estas enfermeiras, não tiveram formação para conhecerem as TA/C e assim, poderem usá-las, recomendá-las, distingui-las dentre as que realmente contribuem no atendimento e sem riscos à população.
Foram levantadas as experiências com as TA/C que as enfermeiras têm consigo mesmas, com seus familiares, usuários/clientes e funcionários de sua unidade de saúde e outros que as procuram.

Quanto à aplicação em si próprias, mais da metade, ou seja, $10(55,6 \%)$ não responderam a esta questão, o que mostra, talvez, uma certa resistência em aceitar e adotar estas práticas. De uma forma coerente, verificamos que $12(66,7 \%)$ não responderam, também, as questões relativas às aplicações em seus familiares $15(83,3 \%)$, em usuários e funcionários e 16(89,9\%) em outros (Figura 2).

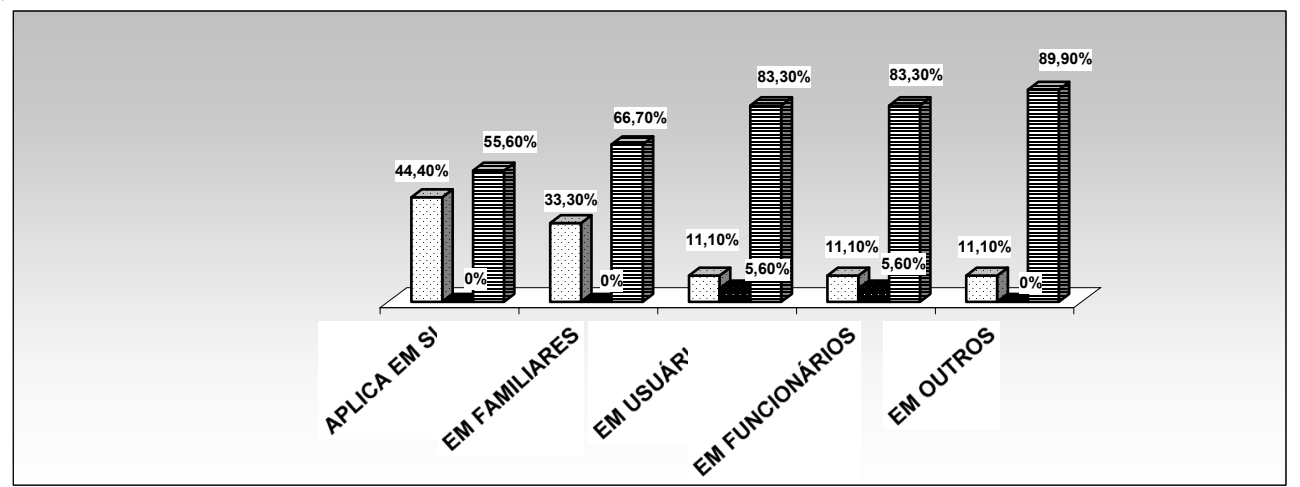

Figura 2: Fazer das enfermeiras do DA 71, em relação às TA/C (São Paulo jun/ago- 2001) 
Helena Maria F. Nuñez Suely Itsuko Ciosak
Oito(44,4\%) enfermeiras mencionaram usar para si mesmas: meditação, fitoterapias com chás, alho, limão, própolis, recebiam acupuntura, do-in, florais de Bach, homeopatia, dietoterapia, e alguns tratamentos naturais; $6(33,3 \%)$ enfermeiras utilizam para seus familiares: meditação, chás de plantas, com alho, cebola, limão, maracujina, capim santo, chá de chuchu, do-in, florais, homeopatia, dietoterapia e alguns tratamentos naturais; $2(11,1 \%)$, enfermeiras aplicam em usuários e clientes da unidade: do-in e dietoterapia em gestantes e alguns tratamentos naturais; $2(11,1 \%)$ empregam em funcionários a dietoterapia, alguns tratamentos naturais e Lian Gong, e para outros que as procuram $2(11,1 \%)$ enfermeiras utilizam: chás de erva cidreira, capim santo, hortelã, dietoterapia e alguns tratamentos naturais.
Sintetizando o fazer das enfermeiras e, considerando a Classificação de Hill( ${ }^{(6)}$, verificamos que as TA/C foram citadas de formas variadas. Averiguamos, também, que algumas enfermeiras utilizavam mais de uma prática. Pudemos notar que, foram mencionadas no total, nove diferentes práticas de TA/C: acupuntura, florais de Bach, do-in, meditação, dietoterapia, ervas medicinais, Lian Gong, (homeopatia e "alguns tratamentos naturais", não foram classificados nos grupos de $\mathrm{TA} / \mathrm{C}$ ), fazendo parte de um total de cinco grupos distintos de TA/C, ou sejam: terapias físicas (meridianas), mentais e espirituais, fitoterapia, nutrição e de exercícios individuais. (Tabela 2).

Tabela 2 - Relação de Grupos de TA/C na classificação de Hill e total de enfermeiras do DA-71 que as aplicam (São Paulo jun/ago- 2001)

\begin{tabular}{|c|c|c|c|c|c|}
\hline \multicolumn{2}{|c|}{$\begin{array}{l}\text { Total de Enfermeiras } \\
\text { que aplicam*: }\end{array}$} & $\begin{array}{l}\text { Terapias alternativas } \\
\text { complementares }\end{array}$ & $\%$ & $\begin{array}{c}\text { Classificação de Hill das } \\
\text { TA/C }\end{array}$ & $\%$ \\
\hline $\mathrm{A}, \mathrm{A}=$ & 2 & Acupuntura & 9,1 & $\begin{array}{l}\text { Fitoterapia } \\
\text { Florais e ervas medicinais }\end{array}$ & 36,4 \\
\hline $\mathrm{A}, \mathrm{A}, \mathrm{B}=$ & 3 & Florais & 13,6 & & \\
\hline $\mathrm{A}, \mathrm{B}, \mathrm{C}=$ & 3 & Do-In & 13,6 & Nutrição & 27,3 \\
\hline $\mathrm{A}, \mathrm{B}=$ & $\mathbf{2}$ & Meditação & 9,1 & & \\
\hline $\mathrm{A}, \mathrm{B}, \mathrm{C}, \mathrm{C}, \mathrm{D}, \mathrm{E}=$ & 6 & Dietoterapia & 27,3 & $\begin{array}{l}\text { Terapias físicas: meridianas: } \\
\text { Acupuntura e Do-In }\end{array}$ & 22,7 \\
\hline $\mathrm{A}, \mathrm{A}, \mathrm{B}, \mathrm{B}, \mathrm{E}=$ & $\mathbf{5}$ & $\begin{array}{l}\text { chás, alho, cebola limão de } \\
\text { erva cidreira, hortelã, } \\
\text { capim santo, Maracujina, } \\
\text { Própolis } \\
\text { como antibiótico natural }\end{array}$ & 22,8 & Terapias Mentais e espirituais & 9,1 \\
\hline$D=$ & 1 & Lian Gong & 4,5 & $\begin{array}{l}\text { Terapia de exercicios } \\
\text { individuais }\end{array}$ & 4,5 \\
\hline $\begin{array}{r}\text { Total Cla } \\
22 \mathrm{Me}\end{array}$ & & $\begin{array}{l}\text { Total Classificado: } \\
7 \text { diferentes práticas }\end{array}$ & 100 & $\begin{array}{c}\text { Total } \\
5 \text { Grupos de TA/C }\end{array}$ & 100 \\
\hline
\end{tabular}

Aplicam*:A= si mesma ; $\mathrm{B}=$ familiares; $\mathrm{C}=$ usuários/clientes da unidade de saúde; $\mathrm{D}=$ funcionários e $\mathrm{E}=$ outros que aprocuram.

Nos dados encontrados por Barbosa ${ }^{(8)} \mathrm{e}$ Souza $^{(5)}$, podemos observar que os quatro grupos de TA/C de maior incidência, utilizadas pelas enfermeiras também foram, em ordem decrescente, a Fitoterapia, Nutrição, Terapias Físicas-meridianas e Terapias Mentais e Espirituais, reforçando os dados desta pesquisa.

Ao conceituar TA/C, 11(61,1\%) das enfermeiras afirmaram que "é um tratamento": ministrado ao paciente sem medicamentos tradicionais, que o profissional pode utilizar junto ou não à medicina propriamente dita, com terapias complementares ;qualquer ação de intervenção natural ou comportamental nos hábitos, que verifiquem na alimentação, repouso, lazer, ou em todas as necessidades humanas básicas; e tem como objetivos a cura, a melhora da qualidade de vida ou minimizar o sofrimento dos pacientes. 
A maioria, ou seja, 15(83,3\%) mesmo sem ter experiência com $\mathrm{TA} / \mathrm{C}$, apoiam a sua prática, principalmente: a dietoterapia, fitoterapia, acupuntura, do-in, florais de Bach, toque terapêutico e a meditação.

Poucas foram as facilidades e muitas as dificuldades encontradas para aplicar TA/C, sendo que: $11(61,1 \%)$ descreveram sobre facilidades aquelas relacionadas à aquisição de ervas medicinais, demanda espontânea para atendimento com $\mathrm{TA} / \mathrm{C}$, oportunidade de maior aproximação com o usuário/ cliente $\mathrm{e}$ favorecimento da introdução das TA/C na unidade de saúde, por ser pública.

Quinze $(83,3 \%)$ referiam dificuldades para exercer as TA/C, relacionadas em quatro grupos: quanto a recursos físicos e humanos da unidade de saúde: espaço físico restrito, escassez de enfermeiras para demanda de pacientes na unidade, falta de credibilidade nas TA/C; capacitação: pouquíssimo material escrito, de pesquisa e audio-visual, falta de cursos e reciclagem, e desconhecimento dos profissionais de saúde; equipe de trabalho: não aceitação da prática por enfermeiros, chefia, médicos, necessidade de ter aprovação da Diretoria, falta de contribuição, pouca disponibilidade de tempo e quanto aos usuários/clientes da unidade de saúde: falta de conscientização e dilvulgação para a população em geral, baixa compreensão, pouca crença, relacionar TA/C com fatos sobrenaturais. Estes dados reforçam os achados de Barbo$\mathrm{sa}^{(8)}$, que relatou as facilidades e dificuldades encontradas pelos enfermeiros ao utilizarem as $\mathrm{TA} / \mathrm{C}$, referindo-se ao

tríduo apoio-aceitação-rejeição, tanto institucional quanto da equipe de trabalho destes profissionais, falta de amparo legal, e ausência de disciplinas oficializadas nos currículos das escolas.

A investigação mostrou o quão grande é o interesse da enfermeiras sobre as TA/C, e o quanto se encontram limitadas pela falta de informação e formação.

Realizar esta investigação foi gratificante, pois o fato de estar averiguando o saber e o fazer das enfermeiras na área das $\mathrm{TA} / \mathrm{C}$, despertou em muitas delas o sentimento de utilidade, interesse em ampliar seus conhecimentos e aplicá-los para melhorar a qualidade de vida individual e da comunidade.

\section{CONCLUSÕES}

Tendo em vista os objetivos propostos os resultados obtidos com a investigação das 18 enfermeiras, lotadas nas unidade de saúde municipais do DA-71, permitem as seguintes conclusões:

1. na população predomina o sexo feminino $(94,4 \%) ; 83,3 \%$ referem ter uma filosofia de vida ou religião, $66,7 \%$ das enfermeiras graduaram em Escolas de Enfermagem privadas; frequentaram um total de 27 cursos de pós graduação; $94,4 \%$ possuem faixa etária acima dos 35 anos; $77,8 \%$ se formaram há mais de 15 anos, 72,2\% têm mais de dez anos de trabalho no Serviço Público, e 61,1\% atuam há menos de cinco anos na unidades de saúde atuais. Essa diversidade de características não influenciou no saber e fazer nas TA/C.

2. quanto aos grupos de TA/C conhecidas e utilizadas, foram: $36,4 \%$ Fitoterapia (Florais de Bach, ervas medicinais); 27,3\% Nutrição (inespecífica); $22,7 \%$ Terapias físicasmeridianas (Acupuntura e Do-In); 9,1\% mentais e espirituais (Meditação) e 4,5\% Terapias de exercícios individuais (Lian Gong).

3. quanto aos resultados obtidos com a aplicação das TA/C: $89 \%$ acreditam nas suas ações; 44,4\% têm experiência; somente 22,2\% conhecem a legislaçãopertinente; $94,5 \%$ não fizeram nenhum curso relacionado; $89 \%$ não conhecem cursos que a tornam especialistas; 44,4\% aplicam-na em si mesmas; 33,3\% aplicam em seus familiares; $11,1 \%$ a aplicam em: usuários/ clientes, funcionários e em outros que as procuram.

4. As dificuldades apontadas por $15(83,3 \%)$ enfermeiras foram: a falta de capacitação em $\mathrm{TA} / \mathrm{C}$, tabus e preconceitos das equipe e dos usuários de sua unidade e recursos físicos.

\section{CONSIDERAÇÕES FINAIS}

Nogueira $^{(7)}$ colocou, há quase 20 anos, que a enfermeira é um"profissional que vem sendo subutilizado pelos sistemas de assistência primária. Sua função terapêutica ainda não é bem aceita. Uma das providências necessárias é que as enfermeiras reconheçam a importância de sua atuação e aceitem o seu novo papel e redefinam suas ações nos serviços de saúde".
Terapias alternativas/ complementares: o saber e o fazer das enfermeiras do

Distrito Administrativo 71 - Santo Amaro São Paulo 
Helena Maria F. Nuñez Suely Itsuko Ciosak
Essa situação ainda não se reverteu. A enfermeira deve abrir este novo espaço, amparar-se com o respaldo legal, associar-se a entidades, participar de formação nesta área. A população tem utilizado muitas TA/C para sua saúde, e pelas dificuldades em encontrar nos serviços de saúde um atendimento integral, cada vez mais, têm buscado outros terapeutas, nem sempre profissionais da saúde, para sanar seus problemas. As enfermeiras desta investigação mostraram-se interessadas nas TA/C,sendo que a maioria acredita que elas podem auxiliar na melhoria da saúde, porém o desconhecimento da legislação, a falta de capacitação teórico- prática, as limita na experiência.

Concordamos com Dra Taka Oguisso que, em uma de suas aulas na disciplina de História da Enfermagem, coloca com propriedade: "precisamos ser enfermeiras capazes de ousar", para buscar o saber e ocupar os espaços com o fazer, efetivo, científico e consciente, pois nós somos enfermeiras agentes ativas da transformação e do desenvolvimento da Enfermagem deste milênio.

\section{REFERÊNCIAS}

(1) Silva MJP, Gimenes OMPV. Florais: uma alternativa saudável- pesquisas revelam tratamentos e resultados dessa terapia. São Paulo: Gente;1999.

(2) Almeida MCP, Rocha JSY. O saber de enfermagem e sua dimensão prática São Paulo: Cortez;1986

(3) Gerber R. Medicina vibracional: uma medicina para o futuro. São Paulo:Cultrix;1988.

(4) Barbosa MA. A Utilização de terapias altenativas por enfermeiros brasileiros, [tese] São Paulo (SP): Escola de Enfermagem da USP;1994.

(5) Souza VT, Enfermeiros que trabalham com terapias complementares: conhecendo sua prática. [Dissertação] São Paulo (SP): Escola Paulista de Medicina da UNIFESP; 2000.

(6) Hill A, organizador. Guia das Medicinas alternativas: todos os sistemas de cura natural.São Paulo: Hemus; [ s.d.].

(7) Nogueira MJC. Fitoterapia popular e enfermagem comunitária. [livre docência ].São Paulo (SP): Escola de Enfermagem da USP; 1983.

(8) Barbosa MA, Egry EY, Queiroz VM. Reflexões sobre a mudança de paradigmas e a adoção das terapias alternativas no Brasil no século XX. Texto Contexto Enferm, 1993; 2 (2):33-44.
(9) Conselho Regional de Enfermagem de São Paulo. Documentos básicos de Enfermagem: principais leis e resoluções que regulamentam o exercício profissional de Enfermeiros, Técnicos e Auxiliares de Enfermagem. São Paulo: Escrituras; 2001.

(10) Antunes MJM. O Enfermeiro e a integralidade da assistência de enfermagem na rede básica do Sistema Único de Saúde. [dissertação], Belo Horizonte (MG): Escola de Enfermagem, Universidade Federal de Minas Gerais; 1996.

(11) Bezerra ALQ. O contexto da educação continuada em enfermagem na visão dos gerentes de enfermagem e enfermeiros d e educação continuada. [tese] São Paulo (SP): Escola de Enfermagem da USP; 2000.

(12) Oguisso T. A mulher na força de trabalho: o trabalho da mulher enfermeira.Rev Enferm UERJ 1998; 6 (1): p 309-16

(13) Oguisso T. Assistência primária de saúde no INAMPS em São Paulo e no Rio de Janeiro: contribuiçãodo enfermeiro. [tese] São Paulo (SP): Faculdade de Saúde Pública da USP; 1984.

(14) Ciosak SI. Avaliação de desempenho e o aprimoramento de enfermeiros: expectativas do avaliador e do avaliado. [tese] São Paulo (SP): Escola de Enfermagem da USP; 1994.

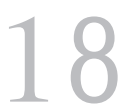

Rev Esc Enferm USP 2003; 37(3): 11-8. 